\title{
Análisis del riesgo \\ crediticio bancario en \\ la economía cubana
}

DOI: 10.22403/UQROOMX/TYP03/06

inthen

\section{RESUMEN}

Zulma María Ledesma Martínez Inocencio Raúl Sánchez Machado*

En este trabajo se presenta un estudio bibliográfico con el objetivo de elaborar el marco teórico o referencial para el análisis del riesgo crediticio bancario en las condiciones concretas de la economía cubana, conforme a las tendencias más actuales en este campo.

Se aborda el estudio del uso de una herramienta estadísticomatemática para robustecer la toma de decisiones respecto del análisis del crédito bancario, combinando las técnicas del análisis económico-financiero tradicional y otras más sofisticadas, con ayuda de herramientas estadístico-matemáticas. Concretamente, el uso de ratios financieros y las técnicas de clustering, árbol de decisiones y el método de Brown \& Gibson para establecer una diferencia entre clientes de acuerdo y su capacidad de pago, valorando la decisión en sus aspectos cualitativos y cuantitativos.

Palabras |Cuba, análisis de riesgo crediticio bancario, árbol de decisiones,

CLAVE clustering, economía cubana, método de Brown \& Gibson.

* Universidad Central de Las Villas Centro de Estudios Turísticos / raulsm@gmail.com 


\section{Introducción}

Es importante dominar las diferentes técnicas de análisis de crédito y complementarlas con una buena cuota de experiencia y buen criterio, así como contar con la información necesaria y suficiente que permita minimizar el número de incógnitas y así poder tomar una decisión correcta y robustecerla.

¿Cómo aplicar diversas técnicas estadístico-matemáticas con el fin de analizar y mejorar la gestión de créditos dentro del sistema bancario cubano?

Se parte de la siguiente hipótesis: si se realizan análisis de carácter cuantitativo y cualitativo del crédito bancario, científicamente fundamentados en técnicas estadístico-matemáticas de avanzada a escala mundial, como el análisis de clustering y método de Brown \& Gibson, se logrará robustecer la toma de decisiones y reducir el riesgo en el análisis y la gestión, mejorando la definición de estrategias y políticas crediticias bancarias.

Para realizar el análisis estadístico se usó el paquete estadístico-matemático SPSS/PC, haciendo uso de las variables discretas.

\section{Una breve introducción teórica al análisis del riesgo del crédito bancario}

Es de vital importancia para la economía cubana, insertada en la región caribeña, que sus empresas, instituciones y la banca, dominen los riesgos asociados al sistema de pagos, con el propósito de controlar y minimizar las consecuencias negativas que éstos provocan $y$, de esta forma, lograr que las operaciones financieras se realicen correctamente.

En el caso particular de las entidades financieras, donde una de las funciones principales es otorgar créditos a otras empresas e instituciones, la elección de clientes se hace totalmente indispensable para garantizar sus operaciones más inmediatas, sin riesgo de incumplimiento ante sus acreedores.

\section{Definiciones para el}

\section{análisis del riesgo de crédito}

El riesgo puede definirse como la oportunidad o probabilidad del surgimiento de algún evento desfavorable. Está ligado a la incertidumbre que rodea en general a cualquier hecho económico, en el sentido de contingencias que puedan 
ocasionar pérdidas.Viene a ser el lado opuesto de la seguridad. El riesgo debe aumentar con el plazo de tiempo.

También se acepta el criterio de riesgo como la contingencia o eventualidad de un daño o de una pérdida como consecuencia de cualquier clase de actividad, y cuyo aseguramiento puede ser objeto de contrato.

Existe la posibilidad de que los clientes de una empresa o institución financiera desarrollen una actitud caracterizada por cualquiera de los siguientes eventos estadísticos:

- Pagar las deudas en el momento concertado en la venta a crédito (clientes dignos).

- No pagar las deudas en el momento concertado en la venta a crédito (clientes no dignos).

Puede ocurrir que los clientes que no liquidaron una venta a crédito en el momento convenido decidan hacerlo con cierto retraso (clientes morosos) antes que ser un cliente no pagador bajo cualquier circunstancia; por eso es aceptable asumir que son tres las actitudes que se pueden verificar en la figura I. Como se podrá observar, la decisión de otorgamiento del crédito en la venta de una entidad estará determinada por la capacidad de cumplimiento de las obligaciones de los clientes, es decir, de la disciplina de éstos a la hora de efectuar sus pagos.

Si se desea aplicar la teoría descrita al problema concreto que se abordó, el análisis de las cuentas por cobrar, tomando en consideración las diferentes situaciones que se acaban de señalar, gráficamente se describiría como lo muestra la figura I:

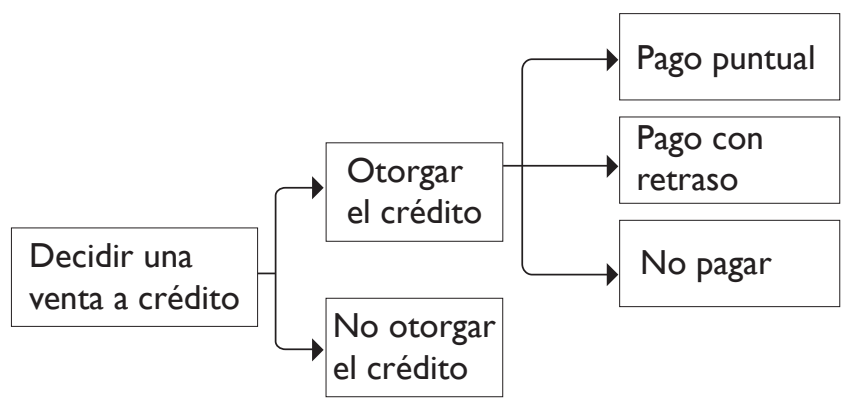

Figura I. DeCisión de otorgamiento de CRÉdito 
Tomando en consideración otros criterios de diversos autores, se puede admitir el siguiente tipo de valoración. Hay dos grandes clases de actitudes de un cliente en el momento contractual de liquidar una venta a crédito:

I. Pagar en tiempo o puntual.

2. No pagar puntualmente.

Ésto, gráficamente se puede representar en la figura 2 y hay que considerar que no se trata de eventos mutuamente excluyentes sino que tienen determinada relación; por ello puede ocurrir un área sombreada según la cual, pueden ser calificados los clientes como no pagadores y también como pagadores (pero con retraso):

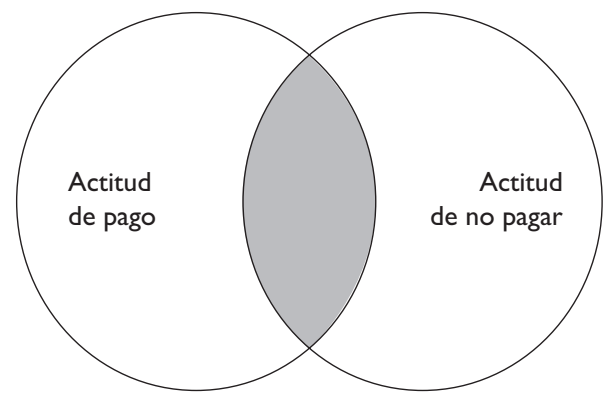

Figura 2. Actitudes de Un CLIENTE

El área sombreada de la figura 2 representa los casos de pago con retraso, el cual es finalmente una disposición de liquidar una deuda pero también de no pagar en el momento debido.

Como el retraso en el pago implica un desfase respecto al pago puntual es válido aceptar el criterio de considerar como eventos posibles los pagos puntuales, pagos con retraso y no pago.

Se parte del supuesto de que un mes es el período en el cual el universo poblacional objeto de estudio, es decir, los clientes, pueden haber adoptado tres actitudes frente al pago de una venta a crédito, aspecto que es factible para la actividad de servicios de la empresa que será ajustada al análisis. Para cualquier análisis económico financiero reviste vital importancia, además, el tratamiento que se le da a la intercompensación entre el riesgo y el rendimiento. 
Toda la teoría financiera y su estudio plantea una hipótesis y la prueba acerca de que a mayor riesgo, mayor rendimiento $y$, al realizar un análisis del crédito financiero, este aspecto también se encuentra latente.

De manera general, entre más alta sea la probabilidad de que el rendimiento real se encuentre por debajo del rendimiento esperado, mayor será el riesgo asociado con el hecho de ser el propietario de un activo.

En el caso particular de este trabajo, se debe tener presente que los conceptos de riesgo y crédito son inseparables. El crédito ideal sería aquel que dé una seguridad total o un riesgo nulo; pero en la práctica, eso es casi imposible: no hay crédito sin riesgo.

Sucede que para las entidades financieras no es suficiente recuperar el dinero prestado, sino que además se debe obtener un rendimiento por las funciones de intermediación y por la asunción del propio riesgo de crédito.

Se establece como condición necesaria recuperar el dinero prestado y como condición suficiente recuperar el dinero + beneficio por la operación. Es decir, al binomio riesgo-crédito habrá que incorporar el concepto de beneficio o rentabilidad de las operaciones de intermediación.

La operación crediticia se desarrolla en la intersección del riesgo-seguridadrentabilidad. Claro está, a menor riesgo, más seguridad pero menos rentabilidad. A menos seguridad, se origina un mayor riesgo y por consiguiente se espera que exista una mayor rentabilidad de la operación crediticia. Es importante reducir el riesgo de una operación crediticia, por lo que su éxito dependerá de si está bien o mal hecha en todas y cada una de sus etapas y fases.

El análisis del riesgo crediticio tiene además dos elementos internos fundamentales: el análisis cuantitativo y el análisis cualitativo, en los que se definirán una serie de variables en dependencia del sujeto de crédito a analizar.

\section{Análisis cuantitativo del crédito}

Esta gestión, como se mencionó anteriormente, puede afrontarse de una forma masiva o adoptando criterios cuantitativos; uno de ellos es a través del uso de razones o ratios financieros.

Una razón o ratio financiero representa una relación entre magnitudes expresadas en unidades monetarias procedentes de la cuenta del balance $y$ del estado de resultado que describe la situación financiera de un negocio. Constituye además una herramienta de análisis y permite cuantificar la relación 
financiera existente entre dos magnitudes y formular un juicio objetivo sobre la solidez, suficiencia o debilidad de dicha relación y comparar la evolución de las empresas.

\section{Análisis de cluster}

Al clasificar a los clientes surgen problemas cuando el analista obtiene ciertas características de una observación o situación y desea clasificarla dentro de una de varias categorías predeterminadas para pormenorizar el análisis. Por ejemplo, un analista financiero tiene a su disposición diversas razones financieras de una empresa y desea usar estas razones para clasificar a la empresa en quiebra o como una empresa no en quiebra. El análisis de cluster es una de las técnicas que permite hacer este tipo de clasificación.

Este análisis, también conocido como análisis de conglomerados, taxonomía numérica o reconocimiento de patrones, es una técnica estadística multivariante, cuya finalidad es dividir un conjunto de objetos en grupos (clustering en inglés) de forma que los perfiles de los objetos en un mismo grupo sean muy similares entre sí (cohesión interna del grupo) y los de los objetos de clusters diferentes sean distintos (aislamiento externo del grupo). En resumen, es una técnica estadística que trata de identificar grupos de objetos o casos similares a partir de un conjunto de atributos.

Es importante comprender que el análisis de cluster no parte-como el análisis discriminante- de un conocimiento previo de los grupos (enfermos o sanos). De hecho, el análisis de clusters clásico no parte siquiera del conocimiento previo de cuántos grupos se deben formar. El análisis de clusters podrá ser usado en combinación con técnicas de análisis discriminante con el objetivo de precisar las reglas de clasificación.

Asimismo, el análisis de clusters clásico es una técnica topológica más que estadística. De hecho, la conformación de grupos se basa apenas en la idea de unir casos o grupos más "cercanos" y por tanto sólo en medidas de distancias entre casos o grupos o medidas de disimilaridad. El objetivo del análisis cluster es obtener grupos de objetos de forma que por un lado los objetos pertenecientes a un mismo grupo sean muy semejantes entre sí, es decir, que el grupo esté cohesionado internamente; $y$, por otro, los objetos pertenecientes a grupos diferentes tengan un comportamiento distinto 
con respecto a las variables analizadas, es decir, que cada grupo esté aislado externamente de los demás grupos.

Conviene enfatizar algunos detalles:

I. El proceso de conformación de clusters jerárquicos puede concebirse como un proceso sucesivo de unión de grupos con un final no premeditado. En principio, el proceso puede hacerse hasta lograr un único cluster y es el investigador quien decide -a partir de criterios de diferentes tipos y que después se analizarán- con cuántos clusters se queda.Tal como lo ilustra la figura 3 los resultados se podrían mostrar del modo siguiente:

*** Hierarchical Cluster Analysis ***

Dendogram using Average Linkage (Between Groups)

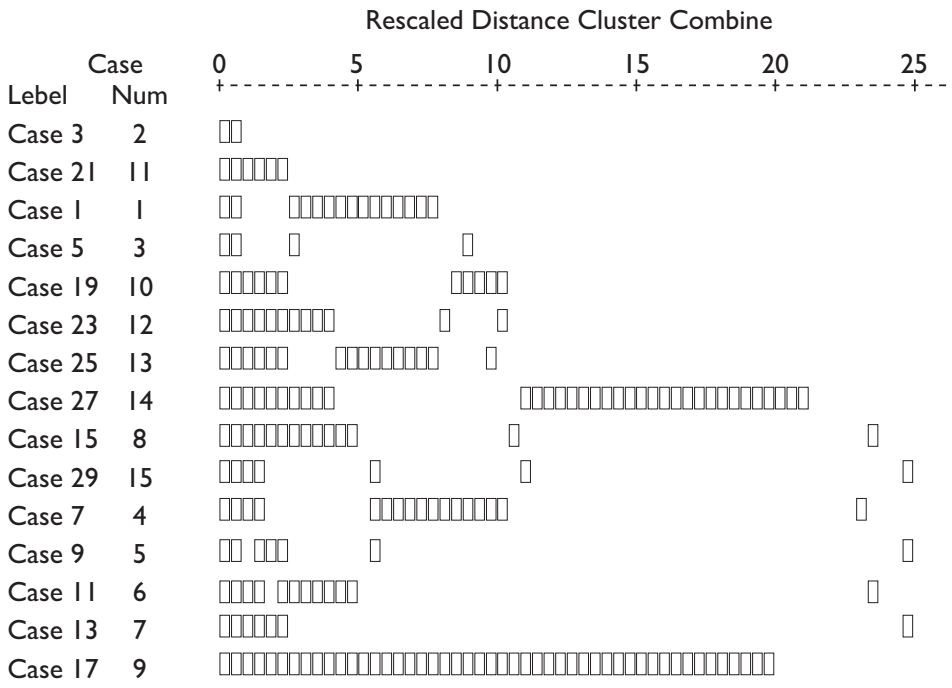

Figura 3. Dendograma del proceso de clustering para el año 2004 
2. El proceso de formación de clusters se define a partir de elecciones importantes:

- Una función de distancia o de disimilaridad de casos.

- Una distancia entre conjuntos o más, precisamente un método de aglomeración de clusters.

Como funciones de distancias puede usarse la euclidiana, la cuadrática euclidiana, la distancia de Manhattan, la distancia potencia ( $p, r)$, la de Chebychev y otras posibles. El SPSS/PC contiene estas distancias y otras como el coseno de vectores basadas en su similaridad. Si no se especifica una distancia, el SPSS/PC trabaja por defecto con la distancia euclidiana. Los métodos de aglomeración de clusters se determinan a partir de la distancia entre conjuntos y se puede contar para ello con cualquiera de las alternativas explicadas y otras más.

\section{Análisis cualitativo del crédito}

El análisis de los aspectos o información cualitativa es otro de los elementos más importantes y al mismo tiempo más difíciles de evaluar de una empresa; su dificultad se debe a que son aspectos subjetivos: la capacidad del ejecutivo, la eficiencia de un sistema gerencial, mecanismos de control de gastos, gestión de ventas, aspectos de producción, instalación, operación, fondos invertidos en asesorías externas, así como la situación de pago de los proveedores: formas de pago de las compras, revisión de los contratos (son importantes porque de ellos depende la gestión de una empresa).

Sintetizando, cualquier debilidad en los factores subjetivos puede tener fatal incidencia en el resultado de la gestión; del mismo modo, cualquier fortaleza en estos factores debe ser detectada y explotada.

- Los insumos: procedencia, transporte y abastecimiento, estabilidad del mercado proveedor, características de la negociación, prestigio de proveedores.

- Los factores externos: dependencia respecto a las políticas económicas, tanto de la empresa como de sus distribuidores y proveedores, mercado internacional, tendencia de la industria, etcétera.

- Producto: calidad, tipos y sus características, dependencia de algún producto especial, sistema de distribución. 
- Las relaciones interempresas: competencia, participación en otras empresas, calidad y tamaño de la competencia, participación de mercado, relación comercial, etcétera.

- El ciclo productivo: este ciclo dependerá del tipo de negocios que fija plazos y condiciones de la organización interna, de su capacidad de proceso productivo, de su nivel tecnológico; estos pueden ser algunos aspectos a tomar en consideración.

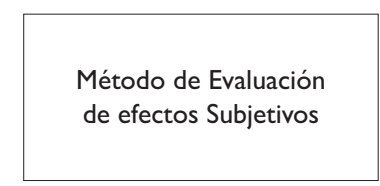

¿Diga qué variables desea incluir?

Comortamiento de los Pagos Administración de la Empresa Situación del Mercado Análisi Sectorial Capacidad Empresarial Tipo de Organiación Otros factores relevantes

¿Diga qué clientes va incluir?

UBPC "La Hoja”

UBPC “Andrés Chongo”

UBPC "Nicolás Fleites"

CAI "Efraín Alfonso"

CAl "I0 de Octubre"

Cultivos Varios "El Diamante"

Empresa de Alimentación Pública

Empresa de Productos Industriales

Empresa de Alimentos

UBPC "Conjedo"

\begin{tabular}{l} 
Comparaciones pareadas \\
Resumen de Variables Subjetivas \\
Variables Objetivas \\
Medida preferencia inversión \\
\hline ¿Diga qué variables económico financieras \\
serán consideradas?
\end{tabular}

0.6

Figura 4. Procedimiento informático de aplicación del Brown \& Gibson para el AÑÁLISIS CUALITATIVO DEL OTORGAMIENTO DE CRÉDITOS 
Uno de los factores de importancia para el éxito del negocio es la experiencia; por ello, se debe saber cuántos años lleva en el negocio, la evaluación de la empresa en cuanto a propiedad y actividad, los hechos más relevantes de su historia. Mientras mayor duración haya tenido la empresa, mayor posibilidad de sobreponerse ante situaciones difíciles; al mismo tiempo podrá aprovechar las experiencias de las situaciones difíciles que haya enfrentado en el pasado.

Debe quedar claro que la administración es clave para la empresa. Si no hay personas competentes, no puede aceptarse crédito.

De igual modo, el gestor de riesgos ha de estar atento a todas las fases del ciclo de la operación: vigilar la definición de límites de riesgo en el momento de las altas de clientes, controlar las condiciones de entrega y facturación del pedido, las incidencias en la facturación y el control de las condiciones y formas de pago al vencimiento. La aplicación de esta herramienta se muestra en la figura 4.

\section{Conclusiones}

En el marco de la gestión financiera operativa, la toma de decisiones de la actividad de inversión financiera tiene un carácter estratégico; la referente a las cuentas por cobrar puede resultar excepcional.La inversión en capital de trabajo es tomada en cuenta para decisiones de largo plazo debido a las repercusiones que tiene en la salud económica financiera de la empresa.

Los niveles de capital de trabajo en la empresa se deciden en un nivel importante por los manejos de los cobros y pagos con que opere una entidad económica de base. La gestión de cobranzas en sus diferentes aristas desempeña un rol decisivo en la reducción de capital inmovilizado y, por consiguiente, en la obtención del máximo rendimiento económico productivo.

En la mayoría de las empresas, así como en el marco de las instituciones financiero crediticias que sirven al proceso de gestión de cobranzas, existe la necesidad de seguir perfeccionando el proceso de cobros y de inversión en tales activos corrientes, con el propósito de racionalizar esta inversión y ordenar de manera prioritaria el otorgamiento de nuevos créditos. La asignación indiscriminada de créditos comerciales y la permisibilidad del aumento de las cuentas por cobrar vencidas, sin un ordenamiento y control efectivos, es un 
problema medular al que hay que brindarle atención priorizada desde una óptica económico-financiera y científicamente tratada en las empresas.

El presente trabajo parte de la aplicación de un procedimiento para mejorar la gestión de cobranzas en las empresas cubanas mediante el empleo de las técnicas del análisis discriminante múltiple por medio del análisis de cluster, sustentado en razones financieras, para contribuir al mejoramiento del desempeño económico financiero de la empresa, particularmente en un aspecto tan importante como la obtención del capital líquido que garantice su funcionamiento. Esto permite discriminar lo relacionado con los retrasos de las cuentas pendientes de cobro y la capacidad de pago de los clientes; para establecer así, condiciones de crédito a los clientes y, por tanto, la elección de políticas de crédito basadas en la diferenciación establecida previamente.

La literatura vigente a nivel mundial acerca del crédito bancario favorece la cobertura para establecer una diferenciación entre clientes acreedores de una entidad bancaria, lo cual facilita y robustece la toma de decisiones y el establecimiento de políticas diferenciadas.

\section{FUENTES CONSULTADAS}

Brealey, R. y Myers, S. (1993). Fundamentos de financiación empresarial. 4a ed. Madrid: McGraw-Hill.

Chao, L. (1993). Estadísticas para las ciencias administrativas. $3^{\text {a }}$ ed. México: McGraw-Hill.

Fernández, A. I. (1994). Introducción a las finanzas. Madrid: Civitas.

Gitman, L. (1997). Fundamentos de administración financiera. $3^{\mathrm{a}}$ ed. Madrid: McGraw-Hill.

Guajardo, G. (1995). Contabilidad financiera. México: McGraw-Hill.

Horngren, C. (1990). Contabilidad administrativa. Introducción. $5^{a}$ ed. México: McGraw-Hill.

Weston, J. (1997). Fundamentos de administración financiera. 10 ed. México: McGraw-Hill. 


\section{$x^{2}$ \\ Observatorio del Turismo}

Universidad de Quintana Roo

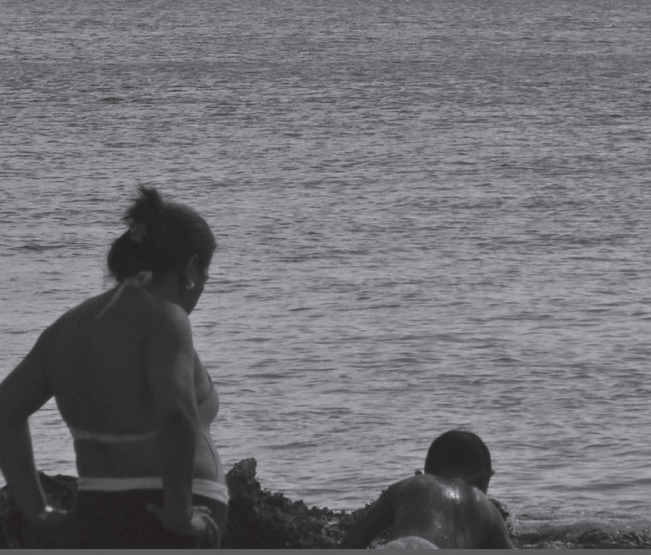

La escasez de información estadística confiable y su dispersión en las entidades publicas o privadas impiden la concentración de información relevante para la toma de decisiones.

Por ello, la División de Desarrollo Sustentable, a través de su Cuerpo Académico de Turismo, crea el Observatorio del Turismo con la finalldad de generar información cuantitativa y cualitativa vinculada a la actividad turística, con el propósito de organizar, fomentar y generar nuevo conocimiento mediante programas de investigación, que tiendan a resolver las necesidades de la sociedad.

Mtro. Romano Segrado Unidad Académica Cozumel 